\title{
Características Anatómicas del Tendón Extensor Accesorio del Dedo Anular, Procedente del Músculo Extensor del Dedo Mínimo
}

\author{
Anatomical Characteristics of the Accessory Extensor Tendon of the \\ Ring Finger, Originating from the Extensor digiti minimi Muscle \\ "de Mendonça Rodas, P. M.; "Rocha, A. C.; "Buarque de Gusmão, L. C.; *Sousa-Rodrigues, C. F. \& "Olave, E.
}

DE MENDONÇA, R. P. M.; RODAS, A. C.; BUARQUE, G. L. C.; SOUSA-RODRIGUES, C. F. \& OLAVE, E. Características anatómicas del tendón del músculo extensor accesorio del dedo anular, procedente del músculo extensor del dedo mínimo. Int. J. Morphol., 24(3):505-508, 2006.

RESUMEN: El músculo extensor del dedo mínimo es una larga y estrecha lámina muscular que se origina en el epicóndilo lateral del húmero, en el tendón extensor común, insertándose, generalmente, a través de dos cintillas tendinosas, en la expansión extensora del dedo mínimo. A veces, emite un tendón accesorio para el dedo anular.El objetivo del presente estudio fue conocer la incidencia, disposición y trayecto de este tendón accesorio. Se estudiaron 60 piezas de miembros superiores, fijadas en formaldehído al $10 \%$, correspondientes a cadáveres de individuos brasileños, adultos, de ambos sexos. El tendón accesorio fue encontrado en 7 manos (11,7\% de los casos). La presencia de ese tendón tiene importancia funcional y clínico-quirúrgica, pues en casos de lesiones en el dorso de la mano que afecten a los tendones del músculo extensor de los dedos, este tendón accesorio podría establecerse como una forma alternativa en la recuperación del movimiento de la extensión del dedo anular. Los resultados complementarán el conocimiento anatómico del conjunto de tendones que constituyen el dorso de la mano.

PALABRAS CLAVE: Mano; Músculos extensores; Tendones; Ortopedia.

\section{INTRODUCCIÓN}

El músculo extensor del dedo mínimo es una cinta muscular fusiforme, parcialmente destacada del músculo extensor de los dedos. Su tendón sigue a través de un compartimiento separado en el retináculo de los músculos extensores, dividiéndose luego en dos cintas tendinosas, donde la más lateral se une al músculo extensor de los dedos. Ambas cintas se unen con la expansión digital dorsal del quinto dedo, teniendo la función de extender la falange proximal del dedo mínimo en la articulación metacarpofalángica y auxiliar a la extensión de las articulaciones interfalángicas, ayudando también en la extensión de la mano, ejerciendo tracción del dedo mínimo (Moore \& Dalley, 2003).

El músculo extensor del dedo mínimo se extiende desde el epicóndilo lateral del húmero a la falange proximal del dedo mínimo, donde su tendón se bifurca generalmente en dos. Este músculo refuerza la acción del músculo extensor de los dedos y contribuye a la relativa independencia de este dedo (Latarjet \& Ruiz-Liard, 1989). El tendón de inser- ción puede no biifurcarse, existiendo la posibilidad de trifurcarse, donde uno de esos tendones va hacia el dedo anular (Williams et al., 1995; Goss, 1988). El tendón para el dedo anular fue mencionado por Testut (1884) y Le Double (1897). En base a estas informaciones, se realizó un estudio sobre este tendón accesorio del músculo extensor del dedo mínimo, para complementar el conocimiento del complejo tendinoso dorsal de la mano, verificando su frecuencia, así como su disposición y trayecto, resaltando así, la importancia clínico-quirúrgica en el área de la ortopedia.

\section{MATERIAL Y MÉTODO}

Se estudiaron 60 miembros superiores formolizados, de individuos adultos, brasileños, de ambos sexos, sin distinción de raza o color, material existente en el Laboratorio de Anatomía Descriptiva y Topográfica del Centro de Ciencias Biológicas de la Universidad Federal de Alagoas

\footnotetext{
* Universidade Federal de Alagoas, Maceió, Brasil.

** Universidad de La Frontera, Temuco, Chile.
} 
(UFAL), Brasil. Las muestras fueron disecadas, exponiendo en el dorso de la mano, los tendones de los músculos extensores, registrando la frecuencia y el trayecto del tendón extensor accesorio del dedo anular, así como su sintopía.

Para sus mediciones se utilizó un caliper mecánico, de marca Metrica. Se midió la longitud del tendón extensor accesorio del dedo anular, la longitud del tendón extensor del dedo mínimo y también se registró su oblicuidad, ya que el tendón accesorio tiene un trayecto oblicuo respecto a los tendones que se dirigen al dedo mínimo. Se esquematizaron y fotografiaron los casos con la presencia del referido tendón.

\section{RESULTADOS}

El tendón extensor accesorio del dedo anular fue encontrado en 7 muestras, o 11,7\% de los casos (Fig. 1b). La formación tendinosa se originó y tuvo una dirección oblicua, a partir de los tendones que van al dedo mínimo. Presentó una íntima relación con la conexión intertendinosa localizada entre el dedo anular y el dedo mínimo, pasando por debajo de la misma.

El vientre del músculo extensor del dedo mínimo era largo, estrecho y fusiforme, llegando sus fibras, hasta un nivel proximal a la articulación radiocarpiana. Su porción terminal presentó una vaina fibrosa propia, presentando cierta oblicuidad para poder fundirse con el tendón que el músculo extensor de los dedos envía al dedo mínimo. Su inervación procedía del nervio interóseo posterior.

El tendón extensor accesorio del dedo anular se presentó más corto que los tendones que van al dedo mínimo, teniendo una longitud promedio de $6,4 \mathrm{~cm}$; en cambio, los tendones que alcanzan el dedo mínimo tuvieron $7,2 \mathrm{~cm}$. Las medidas fueron registradas desde la articulación de la muñeca hasta la falange proximal del quinto dedo.

\section{DISCUSIÓN}

Los tendones son elementos de transmisión de fuerza muscular y como tales, tienen condiciones especiales de funcionalidad, su resistencia a la tracción y su deslizamiento perfecto al lado o en el interior de tejidos y estructuras que los circundan. Cualquier necrosis de un segmento que los debilite o cualquier adherencia que impida su deslizamiento, hará que éstos se tornen inútiles y con frecuencia serán un elemento pernicioso al buen funcionamiento de la mano como un todo (Birolini \& Oliveira, 1985).

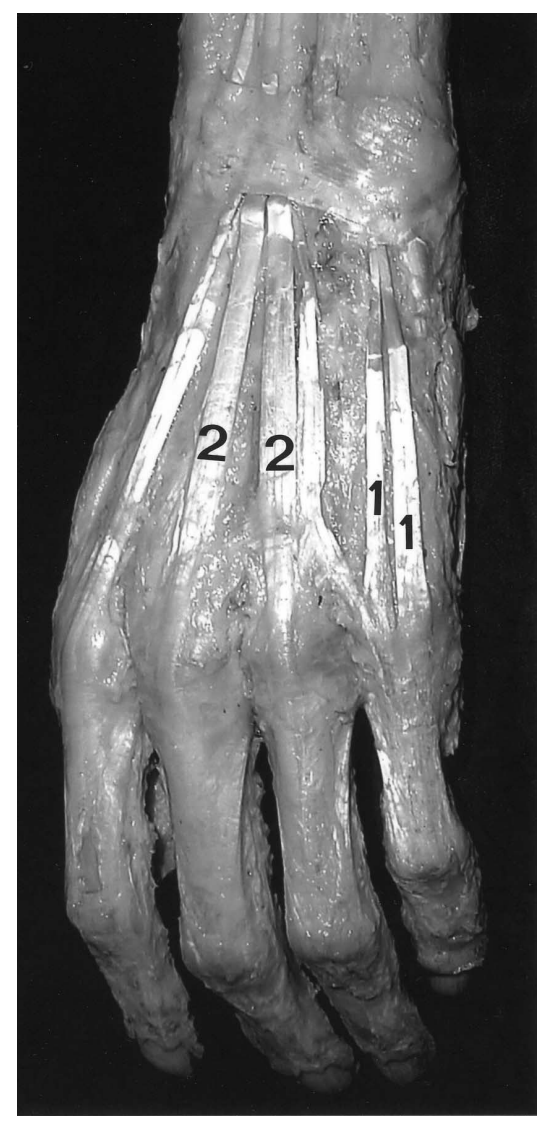

Fig. 1. Dorso de la mano.

1. Cintillas tendinosas del músculo extensor del dedo mínimo; 2. Tendones del músculo extensor de los dedos.

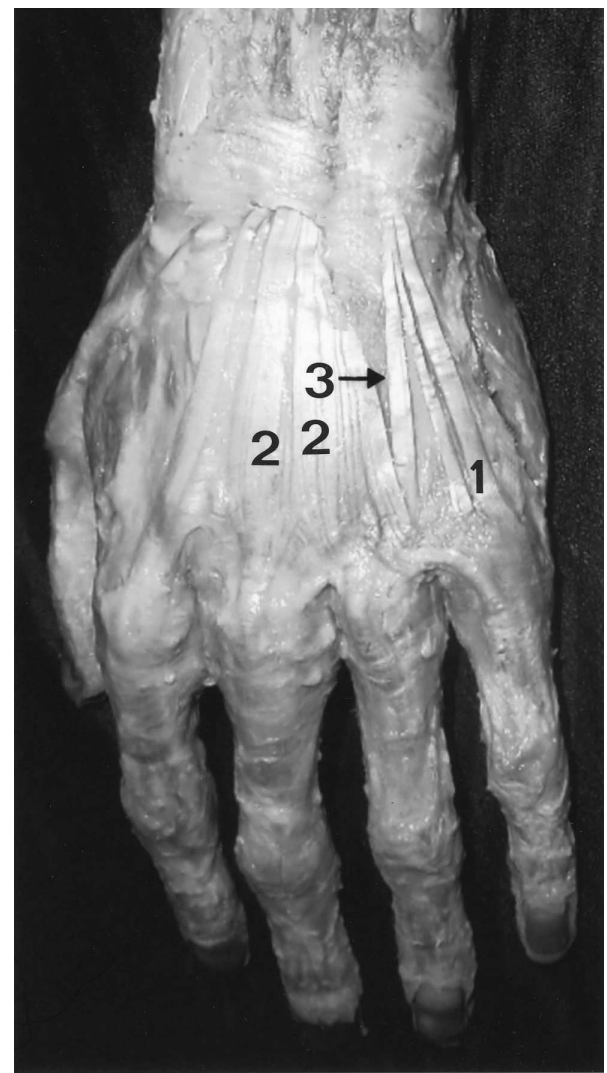

Fig. 2. Dorso de la mano.

1. Cintillas tendinosas del músculo extensor del dedo mínimo; 2. Tendones del músculo extensor de los dedos; 3. Tendón extensor accesorio del dedo anular. 
Se considera que un tercio de los accidentes de trabajo se localizan en las manos y que gran parte de éstos, afectan a los tendones (Goffi, 1996).

Testut, Le Double, Williams et al., Goss y Tountas \& Bergman (1993) fueron los únicos autores en la literatura consultada que se han referido al tendón en estudio, denominándolo simplemente como tendón adicional, aunque este tendón se caracteriza por ser una estructura auxiliar o accesoria para el dedo anular, por lo que optamos llamarlo como tendón extensor accesorio del dedo anular.

En estudios realizados por Testut en 262 muestras, la presencia de este tendón fue confirmada en 26 , lo que equivale a $9,92 \%$ de los casos, resultado un poco menor que el de nuestra serie $(11,7 \%)$. En un estudio realizado en individuos ingleses, Wood (apud Tountas \& Bergman) reportó su presencia en 10,8 \%, valor semejante al encontrado en nuestra serie.

Tanto Williams et al. y Goss comentaron la posibilidad de existencia de un tendón adicional para el cuarto dedo, pero no dan detalles sobre el mismo.

Autores, como Latarjet (1944), Orts-Llorca (1963), Woodburne (1984), Latarjet \& Ruiz-Liard, Gardner (1988), Staubesand (1990), Moore \& Dalley, Spalteholz (1998) no hicieron referencia a ese tendón.

Considerando que el referido tendón se origina a partir del tendón de inserción del músculo extensor del dedo mínimo, las descripciones anatómicas sobre el mismo señalan que se origina desde el epicóndilo lateral del húmero (Moore \& Dalley) y desde el tendón extensor de los dedos, por medio de un fino fascículo tendinoso y a partir de los septos intermusculares adyacentes, originán- dose también de la fascia antebraquial, donde su inserción se realiza por medio de dos cintas tendinosas en el tendon extensor de los dedos y la expansión digital dorsal (Williams et al.) Su ausencia es infrecuente y su tendón de inserción se divide a menudo en dos o más cintillas. $\mathrm{Su}$ vientre muscular puede ser doble (Tountas \& Bergman). Al observar los esquemas del texto de estos últimos autores sobre variaciones musculares, se puede apreciar que cuando existe la cinta tendinosa para el dedo anular se origina de una bifurcación del tendón del músculo extensor del dedo mínimo, a nivel de la línea articular de la muñeca. En los casos que encontramos al tendón accesorio, se originó también a ese nivel, sólo que de una trifurcación.

El gran porcentaje de lesiones en la mano y por ende, en los tendones, como refirió Goffi, resalta la importancia del conocimiento de estos tendones accesorios para ese tipo de lesiones. Cuando se presentan lesiones del músculo extensor de los dedos y existe el tendón extensor accesorio del dedo anular, su importancia es primordial, pues en el caso de que los tendones afectados no sean recuperados total o parcialmente a través de los procesos de sutura o tenorrafia y teniendo en cuenta que la recuperación es lenta, el movimiento de extensión del dedo anular podrá permanecer, aunque no sea con la misma eficiencia.

El estudio realizado permite señalar que el referido tendón extensor accesorio permite una alternativa de movimiento, otorgando un mayor potencial de independencia en la extensión del dedo anular. Por otra parte, la presencia de estos tendones accesorios no debe ser descartada por la anatomía o la clínica, sino despertar la inquietud en la búsqueda de nuevos métodos y conocimientos que tengan la finalidad de perfeccionar las técnicas ya existentes en el área ortopédica y traumatológica

DE MENDONÇA, R. P. M.; RODAS, A. C.; BUARQUE, G. L. C.; SOUSA-RODRIGUES, C. F. \& OLAVE, E. Anatomical characteristics of the accessory extensor tendon of the ring finger, originating from the extensor digiti minimi muscle. Int. J. Morphol., 24(3):505-508, 2006.

SUMMARY: The extensor digiti minimi muscle originates from the posterior aspect of the lateral epicondyle of the humerus from a common tendon for the epicondilar muscles. The muscular belly is long and narrow and runs distally until it joins the ulnar side of a tendon over the radiocarpal articulation. Over the dorsum of the hand, this muscle is represented by a double tendon that insert in the extensor apparatus. Sometimes, these tendons give off an accessory tendon for the ring finger. The objective of this present study was to know the incidence, disposition and course of this accessory tendon. We are study 60 upper limbs from Brazilian adult cadavers of both sexs, fixed in formaldehyde $10 \%$. The accessory tendon of the ring finger was found in 7 hands (11,7\% of the cases). The presence of this tendon have functional and surgical importance; in extensor digitorum tendons injuries, this accessory tendon could be an alternative for recuperate the extension movement of the ring finger. Our results will complete the anatomic knowledge over the dorsal tendons of the hand.

KEY WORDS: Hand; Extensor muscles; Tendons; Orthopedy. 


\section{REFERENCIAS BIBLIOGRÁFICAS}

Birolini, D. \& Oliveira, M. R. Cirurgia do Trauma. Rio de Janeiro, Atheneu, 1993.

Gardner, E. Anatomia, Estudo Regional do Corpo Humano. Rio de Janeiro, Guanabara Koogan, 1988.

Goffi, F. S. Técnica Cirúrgica: Bases Anatômicas, Fisiopatologias e Técnicas da Cirurgia. São Paulo, Atheneu, 1996.

Goss, Ch. M. Gray Anatomia. Rio de Janeiro, Guanabara Koogan, 1988.

Latarjet, A. Tratado de Anatomía Humana. Barcelona, Salvat, 1944.

Latarjet, A. \& Ruiz-Liard, A. Anatomia Humana. São Paulo, Panamericana, 1993.

Le Double. A. F. Traité Des Variatrons du Systeme Musculaire de L'homme. Paris, Academie des Sciences, 1897.

Moore, K. \& Dalley, A. Anatomia Orientada para a Clínica. Rio de Janeiro, Guanabara Koogan, 2003.

Orts Llorca, F. Anatomía Humana. Barcelona, CientificoMedica, 1963.

Spalteholz, W. Atlas de Anatomia Humana. São Paulo, Roca, 1998.
Staubesand, J. Atlas de Anatomia Humana. Rio de Janeiro, Guanabara Koogan, 1990.

Testut, L. Traite Des Variations du Systeme Musculaire de L'homme. Paris, Academie des Sciences, 1884.

Tountas, Ch. \& Bergman, R. Anatomic variations of the upper extremity. New York, Churchill Livingstone Inc., 1993.

Williams, P.; Warwick, R.; Dyson, M. \& Bannister, L. Gray Anatomia. $37^{a}$ ed. Rio de Janeiro, Guanabara Koogan, 1995.

Woodburne, R.T. Anatomia Humana. Rio de Janeiro, Guanabara Koogan, 1984.

Wood apud Tountas, Ch. \& Bergman, R. Anatomic variations of the upper extremity. New York, Churchill Livingstone Inc., 1993.

Dirección para correspondencia:

Prof. Dr. Célio de Sousa Rodrigues

Departamento de Morfologia

Universidade Federal de Alagoas

Maceió - $\mathscr{A L}$

BRASIL

Recibido : 14-03-2006

Aceptado: 25-07-2006 